\title{
Double Embedded Processes Based Hidden Markov Models for Binary Digital Wireless Channels
}

\author{
Omar S. Salih ${ }^{\# 1}$, Cheng-Xiang Wang ${ }^{\# 2}$, David I. Laurenson ${ }^{* 3}$ \\ \# Joint Research Institute for Signal and Image Processing, Heriot-Watt University \\ Edinburgh, EHI4 4AS, UK \\ 1 uss3@hw.ac.uk \\ ${ }^{2}$ cheng-xiang.wang@hw.ac.uk \\ * Joint Research Institute for Signal and Image Processing, University of Edinburgh \\ Edinburgh, EH9 3JL, UK \\ 3 dave.laurenson@ed.ac.uk
}

\begin{abstract}
Generative models hold the promise of reducing the computational load and cost caused by directly simulating a real system. They are vital to the design and performance evaluation of error control schemes and high layer wireless communication protocols. Therefore, designing an efficient and accurate generative model is highly desirable. Moreover, the errors encountered in digital wireless channels exhibit correlation among them. This stimulates us to construct a Markovian based generative model with two embedded processes. The first process is dedicated to assembling error bursts with error-free bursts, whereas the second one is devoted to creating individual error bursts employing the maximum gap norm within error bursts. This premise is utilized in this paper to show that the resulting generative model can generate error sequences with desired bit correlations and is capable of statistically matching a descriptive model, derived from an enhanced general packet radio service (EGPRS) transmission system, regardless of the configuration of its error sequences.
\end{abstract}

\section{INTRODUCTION}

A digital (discrete-time) channel comprises the complete transmission chain in a communication system, including the transmitter, the physical channel, and the receiver normally expressed in the complex baseband. Error models aim to characterize error sequences obtained by comparing input sequences with output sequences of digital channels. Errors perceived in digital wireless channels do not occur separately, but in bursts or clusters. In order to combat these errors, it is vital to develop error models. Error models have two main advantages. First, they are significant to the design of error control schemes and high layer communication protocols [1], where knowledge of the burst error statistics are really beneficial. Second, the underlying digital channels can be simulated by generated error sequences. Therefore, the computational load and time can be greatly reduced. Error models can be classified into two types: descriptive and generative [2]. A descriptive model identifies the statistical behavior of error sequences gained from real communication systems. Whereas, a generative model is a mathematical representation that generates error sequences with similar statistical properties to those produced by a descriptive model.

In the literature, researchers have proposed various generative models which can be classified into five categories.
The first catagory is based on Markov chains to construct Markov models (MMs). The first model within this category is Gilbert-Elliot model [3]. It is composed of only two states, a bad or error state and a good state. The burst error statistics resulting from this model are in general unsatisfactory due to its simplicity. Thereafter, many modifications have been made to improve this model [4], [5] including increasing the number of states like the well-known simplified Fritchman models (SFMs) [6]. They consist of one error state and several good states. MMs have many applications [7], [8]. The second category is Hidden MMs (HMMs) [9]. They have been established to convey complicated error sequences which are identified by long error bursts interweaved with longer error-free bursts. Baum-Welch based HMMs (BWHMMs) give reasonable results for error profiles which have error bursts with a bell-shaped error density [10], [11]. However, there is still room for the HMMs to be further improved, specifically to make them applicable to other configurations of error sequences. Stochastic-Context Free Grammars models (SCFGs) [11] are considered as the third category. The SCFGs have been designed to apply to indoor channels, but they have not succeeded in supplanting HMMs. Chaos theory has been employed to construct the fourth category of generative models. Chaotic models which depend upon chaotic attractors [12] are certainly complex but their results fail to trace the HMMs in many aspects. Chaotic models which rely on chaotic maps [13] exhibit good results for some types of error patterns, particularly heavy-tailed run length error patterns. The last category is the deterministic process based generative models (DPBGMs) [14], [15]. Their idea stems from the second order statistics of fading envelope processes. The DPBGMs are the best generative models in terms of providing realistic burst error statistics. However, a drawback of the DPBGMs is that they do not construct new error bursts in the process of generating error sequences. Instead, they fetch them directly from the original error sequences according to their lengths.

In this paper, we propose a novel generative model, called Double Embedded Processes based Hidden Markov Model (DEPHMM). It consists of two layers of processes. The first layer is composed of only one error-free state and many error 
bursts states. Whereas, the second layer constructs detailed error bursts inside the error bursts states. Estimating the DEPHMM parameters is intuitive and plain. The DEPHMM implementation is simple and the resulting burst error statistics are satisfactory. Binary bit error sequences are considered.

This paper is outlined as follows. Section II introduces some terms and definitions related to the binary bit error sequences and their statistics. Section III reveals the full structure of the DEPHMM and its parametrization. The simulation results of the burst error statistics for the DEPHMM, compared with those of a descriptive model, derived from the EGPRS transmission system, and several other generative models, are displayed in Section IV. Conclusions are drawn in Section V.

\section{TERms AND Definitions}

Suppose a binary input sequence to a digital wireless channel is $\left\{i_{j}\right\}$ and a corresponding binary output sequence is $\left\{o_{j}\right\}$. Note that $i_{j}, o_{j} \in\{0,1\}, j=1,2, \ldots$. The resulting binary error sequence $\left\{e_{j}\right\}$ is obtained by performing modulo 2 addition of the input sequence and output sequence, i.e., $e_{j}=i_{j} \oplus o_{j}$, where $\oplus$ is a modulo 2 addition operator. Clearly, a binary error sequence is a sequence of " 0 "s and " 1 "s, where " 0 " denotes a correct bit and " 1 " denotes an error bit. The error sequence is a fundamental term in error models, hence, requires detailed analysis.

A gap is described as a series of successive zeros between two ones. Its length equals the number of zeros. An error cluster is a series of successive ones having a length equal to the number of ones. To highlight some specific gaps with very long lengths, a threshold value $\eta$ can be assigned to distinguish between these long gaps and the other gaps which are considered as part of the error bursts. This leads us to two new definitions: error-free burst and error burst. An error-free burst is a sequence of successive zeros that have at least $\eta$ bits in length. Note that an error-free burst can in general be considered as a gap with a minimum length restriction, except that an error-free burst is not necessarily located between two ones. An error burst is a sequence of zeros and ones delimited by ones. It is separated from other error bursts by error-free bursts. The minimum length of an error burst is 1. Note that error sequences can be considered as the combination of consecutive error-free bursts and error bursts, or the combination of consecutive gaps and error clusters. On the other hand, error bursts also consist of consecutive gaps with lengths less than $\eta$ and error clusters.

For the purpose of analyzing error sequences comprehensively, many burst error statistics have been elaborated in the literature [2], [15]. These statistics are also used to investigate the performance of generated error sequences. The burst error statistics that will be used in this paper are listed below:

1) $P\left(0^{m_{0}} \mid 1\right)$ : error-free run distribution (EFRD), which is the probability that an error is followed by $m_{0}$ or more error-free bits [6].

2) $P\left(1^{m_{c}} \mid 0\right)$ : error-cluster distribution (ECD), which is the probability that a correct bit is followed by at least $m_{c}$ successive error bits [6].
3) $P_{E B}\left(m_{e}\right)$ : error-burst distribution (EBD), which is the cumulative distribution function (CDF) of error burst lengths $m_{e}$ [15].

4) $P_{E F B}\left(m_{\bar{e}}\right)$ : error-free burst distribution (EFBD), which is the CDF of error-free burst lengths $m_{\bar{e}}$ [15].

5) $P(m, n)$ : block error probability distribution (BEPD), which is the probability that a block of $n$ bits contains at least $m$ errors [2].

6) $\rho(\Delta k)$ : bit error correlation function (BECF), which is the conditional probability that the $\Delta k$ th bit following an error bit is also in error [2].

\section{The PROPOSED Generative Model}

The starting point in configuring the DEPHMM is to divide an obtained reference error sequence from a real system into error bursts and error-free bursts. But, to ascertain the errorfree bursts we firstly need to find out the value of $\eta$. It can be chosen through a range of values between $\eta_{i}$ and $\eta_{f}$ so that the error burst identification is not affected. Both values are simply obtained from the EFRD curve when it is flat.

Fig. 1 shows a sketch of the DEPHMM. Since the error-free bursts consist of only " 0 "s, one state is sufficient to represent them. On the other hand, the error bursts are considered as the most important part in the DEPHMM, because they have many structural variations. Therefore, error bursts deserve to be classified into many groups. Each group should convey a specific structural behavior. Subsequently, a structural criterion must be adopted for the purpose of error bursts classification. The maximum gap in each error burst of the reference sequence is the choice. Explicitly, the maximum gaps of error bursts which could range from 0 to $\eta-1$ should be divided according to their histogram into the number of error burst states, where the divided intervals of the maximum gaps have approximately equal number of error bursts. By using this criterion, the model can circumscribe any degradation in error correlation, which is adequately obvious in wireless communication systems. Joining the error burst states with the error-free burst state to construct generated error sequences is the first process in the DEPHMM.

Producing error-free bursts is straightforward. Once we recognize the reference error-free burst lengths distribution, many error-free burst lengths can then be generated. The big challenge now is how to construct generated error bursts so that they convey the same behavior of the reference error bursts. Because error bursts are composed of error clusters and gaps with lengths less than $\eta$, both of them can be represented by a separate number of states. As a result, many state configurations are possible. But, since our major apprehension is the detailed construction of errors within an error burst, we allot several substates for the error clusters of each class of the first process and single substate for the gaps of the error bursts. Each error cluster substate is occupied by a single error cluster length due to error clusters' short lengths. Similar to the errorfree bursts, the production of gaps within error bursts of each class depends mainly on their length probability distribution. Connecting the error cluster substates with the gaps substate 
is the second process. The mean gap length of the generated error bursts in each state should match that of the original error bursts. Otherwise, the states with mismatched mean gap lengths should be further partitioned.

The parameters of the DEPHMM are as follows:

1) $N$ : the number of states for error bursts, i.e., $S=$ $\left\{s_{1}, s_{2}, \ldots, s_{N}, s_{N+1}\right\}$, where $S$ is the set of states. $N$ is selected according to the accuracy demand.

2) $M_{u}$ : the number of error cluster substates in each state, i.e., $V_{u}=\left\{v_{1 u}, v_{2 u}, \ldots, v_{M u}, v_{M u+1}\right\}$, where $V_{u}$ is the set of substates and $u=1, \ldots, N . M_{u}$ is designated according to the number of error cluster lengths in each error burst state.

3) $\mathbf{F}=\left(f_{i, j}\right)$ : the state transition matrix, where $f_{i, j}$ is the transition probability from $s_{i}$ to $s_{j}$, such that

$$
\begin{gathered}
f_{i, j}=P\left[Q_{t+1}=s_{j} \mid Q_{t}=s_{i}\right], 1 \leq i, j \leq N+1 \\
= \begin{cases}1, & 1 \leq i \leq N, j=N+1, \\
\frac{N_{E B, j}}{\sum_{j=1}^{N} N_{E B, j}} \approx \frac{N_{E B, j}}{N \times N_{E B, N}}, & i=N+1,1 \leq j \leq N, \\
0, & \text { otherwise, }\end{cases}
\end{gathered}
$$

with $Q_{t}$ being the current state at time $t$ and $N_{E B, j}$ the number of error bursts in $s_{j}$. The structure of the state transition matrix is

$$
\mathbf{F}=\left(\begin{array}{cccc}
0 & \cdots & 0 & 1 \\
\vdots & \ddots & \vdots & \vdots \\
0 & \cdots & 0 & 1 \\
f_{N+1,1} & \cdots & f_{N+1, N} & 0
\end{array}\right)
$$

4) $\mathbf{D}_{u}=\left(\left(d_{h, k}\right)_{u}\right)$ : the substate transition matrix, where $\left(d_{h, k}\right)_{u}$ is the transition probability from $v_{h_{u}}$ to $v_{k_{u}}$, such that

$$
\begin{aligned}
& \left(d_{h, k}\right)_{u}=P\left[R_{t+1}=v_{k_{u}} \mid R_{t}=v_{h_{u}}\right], 1 \leq h_{u}, k_{u} \leq M_{u}+1 \\
& \quad= \begin{cases}1, & 1 \leq h_{u} \leq M_{u}, k_{u}=M_{u}+1, \\
\frac{N_{C, k_{u}}}{\sum_{k_{u}}^{M_{u}=1} N_{C, k_{u}}}, & h_{u}=M_{u}+1,1 \leq k_{u} \leq M_{u}, \\
0, & \text { otherwise, }\end{cases}
\end{aligned}
$$

with $R_{t}$ being the current state at time $t$ and $N_{C, k_{u}}$ the number of error clusters in $k_{u}$. The structure of the substate transition matrix is

$$
\mathbf{D}_{u}=\left(\begin{array}{cccc}
0 & \cdots & 0 & 1 \\
\vdots & \ddots & \vdots & \vdots \\
0 & \cdots & 0 & 1 \\
d_{M_{u}+1,1} & \cdots & d_{M_{u}+1, M_{u}} & 0
\end{array}\right) .
$$

5) $\mathbf{A}=\left(a_{j}(n)\right)$ : the first process emission probability distribution matrix, where $a_{j}(n)(1 \leq j \leq N+1)$ is the probability of getting the burst $y_{n}$ in state $s_{j}$, that is

$$
a_{j}(n)=P\left[y_{n} \text { at } t \mid Q_{t}=s_{j}\right], 1 \leq n \leq N_{E B, j}, N_{E F B, j} .
$$

$N_{E F B, j}$ is the number of error-free bursts in $s_{j}$.

6) $\mathbf{B}=\left(b_{k_{u}}(m)\right)$ : the second process gap emission probability distribution matrix, where $b_{k_{u}}(m)\left(k_{u}=M_{u}+1\right)$ is the probability of getting the gap $x_{m}$ in state $v_{k_{u}}$, that is

$$
b_{k_{u}}=P\left[x_{m} \text { at } t \mid R_{t}=v_{k_{u}}\right], 1 \leq m \leq N_{G, k_{u}} .
$$

$N_{G, k_{u}}$ is the number of gaps in $k_{u}$.

7) $\Pi_{u}=\left(\left(\pi_{k}\right)_{u}\right)$ : the initial substate distribution vector, where $\left(\pi_{k}\right)_{u}$ is the probability of $v_{k_{u}}$ to be an initial substate.

$$
\boldsymbol{\Pi}_{u}=\left(d_{M_{u}+1,1}, \ldots, d_{M_{u}+1, M_{u}}, 0\right),
$$

which is extremely important since it assures the initiation of an error burst by an error cluster, otherwise the definition of error burst is no longer valid. By analogy, we can obtain the initial state distribution vector.

8) $\boldsymbol{\Omega}_{u}=\left(\left(\omega_{k}\right)_{u}\right)$ : the termination substate distribution vector, where $\left(\omega_{k}\right)_{u}$ is the probability of $v_{k_{u}}$ to be the final substate.

$$
\boldsymbol{\Omega}_{u}=\left(d_{M_{u}+1,1}, \ldots, d_{M_{u}+1, M_{u}}, 0\right),
$$

which is also very important because it ensures that the error burst is finalized with an error cluster as specified in the definition.

9) $\delta_{n, u}$ : error burst length values. These values regulate the termination of error bursts generation, so that $\Omega_{u}$ shall be activated according to them. The Activation takes place when the generated error burst lengths become either equal or around a chosen $\delta_{n}$. The deviation from $\delta_{n}$ should be small enough, otherwise, the current generated error burst will be discarded. $\delta_{n, u}$ are acquired from the reference error burst length distribution.

10) $\Gamma$ : generated error sequence length. This value terminates the error sequence generation once it is reached or exceeded. It does not matter whether the current state is error burst or error-free burst.

All the aforementioned parameters construct the DEPHMM and explain its algorithm.

\section{Simulation Results and Discussions}

In order to scrutinize the DEPHMM operation, a reference error sequence needs to be obtained for its parametrization. To achieve this, we consider an uncoded EGPRS transmission system with ideal frequency hopping. The elementary digital channel is constituted of a Gaussian minimum shift keying (GMSK) modulator, an interfered propagation channel, a GMSK demodulator, and a hard decision Viterbi equalizer. The underlying channel is tailored to a typical urban (TU) environment with carrier-to-interference ratio (CIR) of $8 \mathrm{~dB}$, and mobile speed of $3 \mathrm{~km} / \mathrm{h}$. The data are transmitted using time-division multiple access (TDMA) with blocks of 116 bits and a transmission rate of $F_{s}=270.8 \mathrm{~kb} / \mathrm{s}$

The reference error sequence has 15 million bits. It exhibits long error bursts interweaved with long error-free bursts. It has 4269 error bursts and 4268 error-free bursts with maximum lengths of 6489 and 6251 bits, respectively. We find $\eta$ from Fig. 2 which displays the EFRD. From its shape plateau, we 
find $\eta_{i}=400$ and $\eta_{f}=1000$ hold. The chosen value of $\eta$ is 800. $\Gamma=21$ million bits.

For the sake of comparison, a SFM, BWHMM, and DPBGM are implemented. The parameters of a SFM with $K$ states are obtained by fitting the weighted sum of $K-1$ exponentials to the EFRD. The number of states used for the SFM is 6. In fact, no better improvement of the SFM statistics could be attained by increasing its number of states to more than 6. In the BWHMM, the number of classes is 12 , whereas the total number of states is 400 . The number of bits which should represent each block in the error bursts is chosen to be 103 bits. For the DPBGM, the vector $\Psi=$ $(9,10,0.09,0.0783,73.22 \mathrm{~Hz}, 0.8132 \mathrm{~ms}), R_{B}=0.9344$, and $q_{s}=0.01$ hold.

To assess any generative model, we need to investigate how close its burst error statistics can match the descriptive model. Figs. 2-7 illustrate the behavior of the burst error statistics, mentioned in Section II, for the descriptive model and the well-known generative models addressed before. It is apparent that the SFM fails to characterize all the statistics except the EFRD, since the SFM is designed by fitting it. The BWHMM depicts an enhancement to the SFM, despite the fact that it misses the contiguity with the descriptive model. This is because the BWHMM was designed to best describe error sequences with bell-shaped error density bursts. But, our reference error sequence has many error bursts which do not comply with such a shape. However, the DPBGM statistics has small differences from the DEPHMM statistics of high accuracy. Nevertheless, the DEPHMM leads the other generative models since the DPBGM fetches the error bursts from the reference error sequence rather than constructing them by itself. In Figs. 2-5, 10 classes of error bursts are selected for the DEPHMM. However, this number of classes is not sufficient to fit the EFBD and BECF in Figs. 6-7 perfectly. Therefore, we increased this number to 20 and 30. As a result, a notable augmentation in the performance is achieved. Although the total number of states of the DEPHMM reaches 260 (including the substates), it is still less than the number of states of the BWHMM. Similar to the EFBD and BECF, it is worth mentioning that the burst error statistics in Figs. 2-5 could be enhanced as well by increasing the number of classes.

\section{CONCLUSIONS}

This paper has demonstrated a novel method for developing a binary HMM using double embedded processes. By this method, the error bursts can be constructed rigorously and hence the generated error sequence. As a consequence, the correlation properties of the generated error sequence can approximate those of the reference error sequence. The SFM and BWHMM are unable to meet these criteria, whereas small differences have been detected between the DPBGM and DEPHMM. This has been examined by a descriptive model obtained from the EGPRS system. Additionally, the DEPHMM can deal with any formation of error sequences, no matter whether it has a special shape of error bursts or not. Whereas, the BWHMM has good behavior only for error sequences with bell-shaped error density bursts. However, the DPBGM does not construct the error bursts.

\section{ACKNOWLEDGMENT}

The authors would like to acknowledge gratefully the sponsorship of this work by the EPSRC and Philips Research Laboratories (PRL), Redhill, Surrey, UK. They would also like to thank Dr. Tim Moulsley from PRL for helpful discussions.

\section{REFERENCES}

[1] M. Zorzi and R. R. Rao, "Perspectives on the impact of error statistics on protocols for wireless networks," IEEE Personal Commun., vol. 6, no. 10, pp. 32-40, Oct. 1999

[2] L. N. Kanal and A. R. K. Sastry, "Models for channels with memory and their applications to error control," Proc. of the IEEE, vol. 66, no. 7, pp. 724-744, July 1978.

[3] E.O. Elliot, "Estimates of error rates for codes on burst-noise channels," Bell Syst. Tech. J., vol. 42, pp. 1977-1997, Sept. 1963.

[4] G. T. Nguyen, R. Katz, and B. Noble, "A trace-based approach for modeling wireless channel behavior," in Proc. WSC'96, Coronado, CA, USA, Dec. 1996, pp. 597-604.

[5] G. Boggia, D. Buccarella, P. Camarda, A. D'Alconzo, “A simple ON/OFF logarithmic model for frame-level errors in wireless channels applied to GSM,' in Proc. IEEE VTC'04-Fall, Los Angeles, CA, USA, Sep. 2004, pp. 4491-4495.

[6] B. D. Fritchman, "A binary channel characterization using partitioned Markov chains," IEEE Trans. Information Theory, vol. 13, no. 2, pp 221-227, Apr. 1967.

[7] Y. Wang, J. Yin, W. Fu, D.P. Agrawal, "Performance enhancement scheme for wireless LAN under bursty Channel," in Proc. IEEE WCNC'07, Hong Kong, Mar. 2007, pp. 2155-2160.

[8] H.-H. Hung, L.-J. Chen, "An analytical study of wireless error models for bluetooth networks," in Proc. IEEE AINA'08, GinoWan, Japan, Mar. 2008, pp. 1317-1322.

[9] M.V. Santos, E.L. Pinto, M. Grivet, "A new approach to the statistical analysis of HMM modelled bursty channels," Proc. IEEE ICC'04, Paris, France, June 2004, pp. 567-572.

[10] J. Garcia-Frias and P. M. Crespo, "Hidden Markov models for burst error characterization in indoor radio channels," IEEE Trans. Veh. Technol., vol. 46, no. 6, pp. 1006-1020, Nov. 1997.

[11] W. Zhu and J. Garcia-Frias, "Stochastic context-free grammars and hidden Markov models for modeling of bursty channels," IEEE Trans. Veh. Technol., vol. 53, no. 3, pp. 666-676, May 2004

[12] E. Costamagna, L. Favalli, and P. Gamba, "Multipath channel modeling with chaotic attractors," Proc. of the IEEE, vol. 90, no. 5, pp. 842-859, May 2002.

[13] A. Köpke, A. Willig, and H. Karl, "Chaotic maps as parsimonious bit error models of wireless channels," Proc. IEEE INFOCOM'03, San Francisco, USA, Mar. 30-Apr. 3, 2003, pp. 513-523.

[14] C.-X. Wang and M. Pätzold, "A generative deterministic model for digital mobile fading channels," IEEE Commun. Letters, vol. 8, no. 4, pp. 223-225, Apr. 2004

[15] C.-X. Wang and W. Xu, "A new class of generative models for bursterror characterization in digital wireless channels," IEEE Trans. Comm., vol. 55, no. 3, pp. 453-462, March 2007

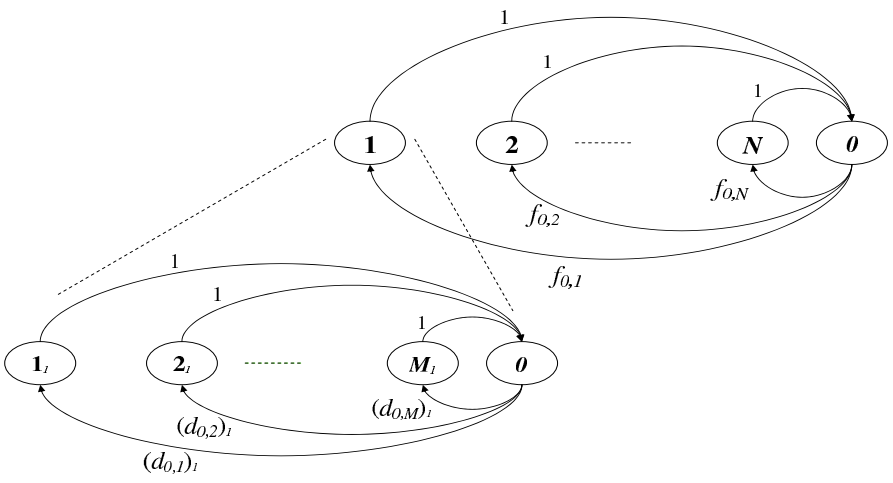

Fig. 1. The DEPHMM. 


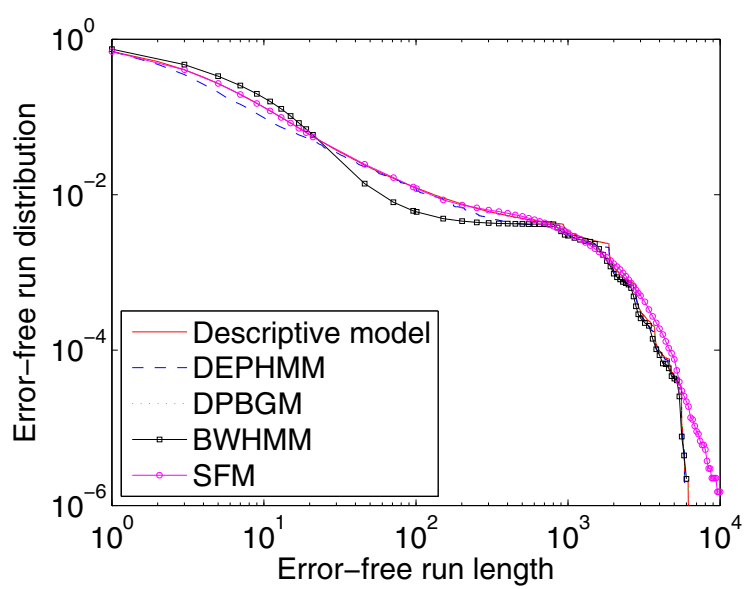

Fig. 2. Error-free run distributions of the descriptive model obtained from the EGPRS system and different generative models.

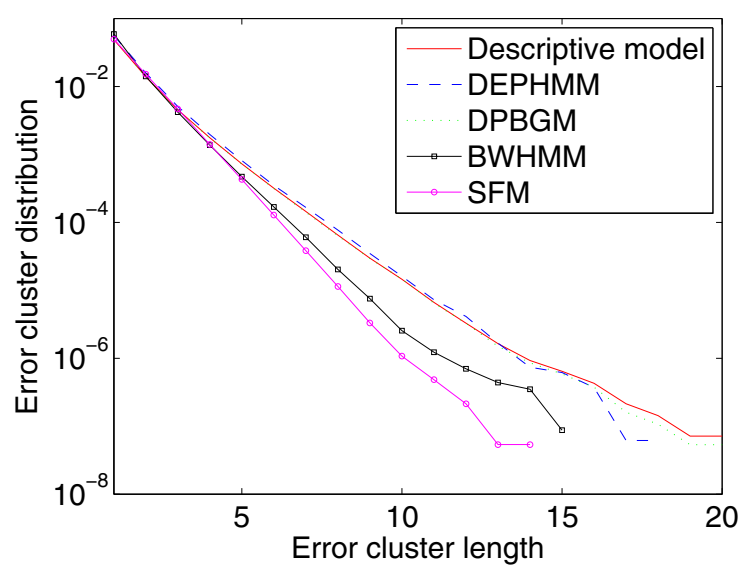

Fig. 3. Error cluster distributions of the descriptive model obtained from the EGPRS system and different generative models.

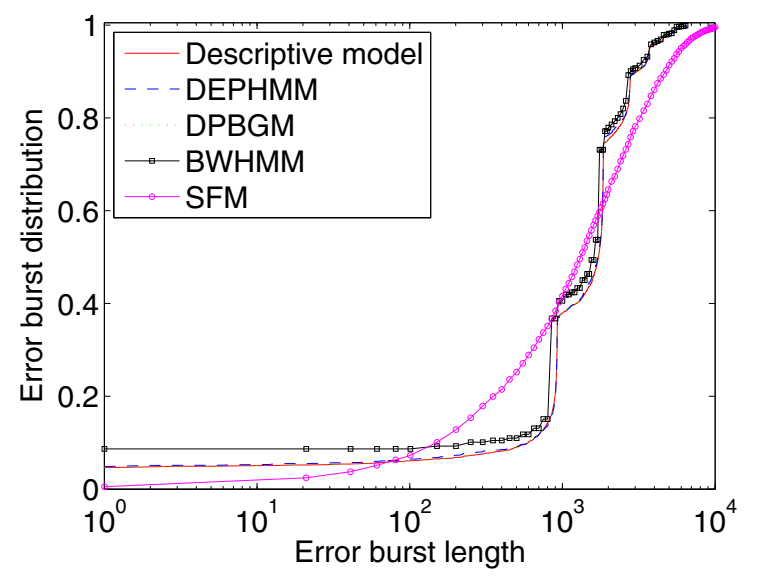

Fig. 4. Error burst distributions of the descriptive model obtained from the EGPRS system and different generative models.

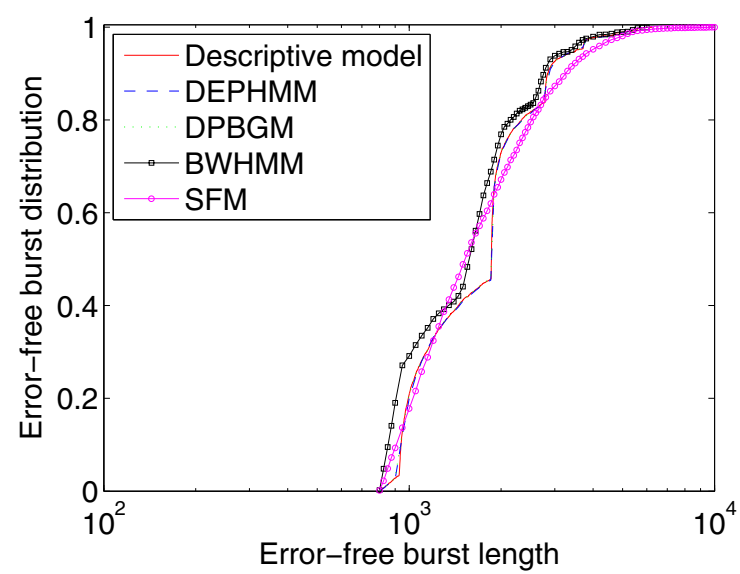

Fig. 5. Error-free burst distributions of the descriptive model obtained from the EGPRS system and different generative models.

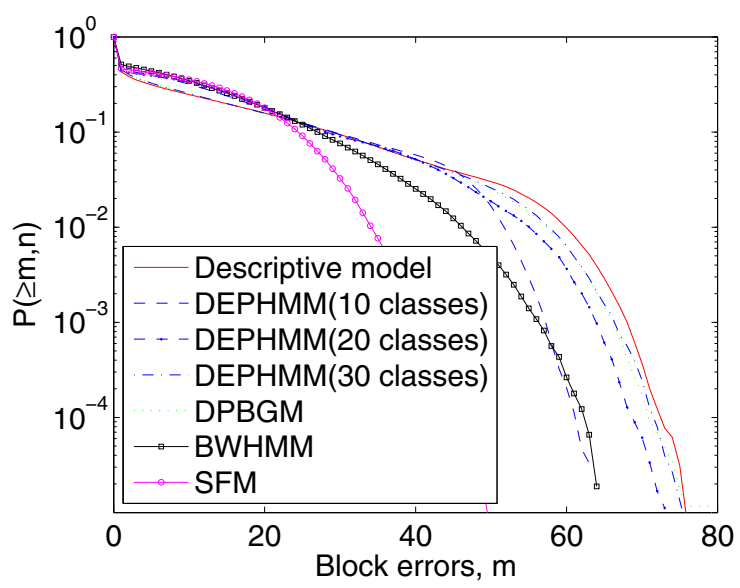

Fig. 6. Block error probability distributions of the descriptive model obtained from the EGPRS system and different generative models $(n=116)$.

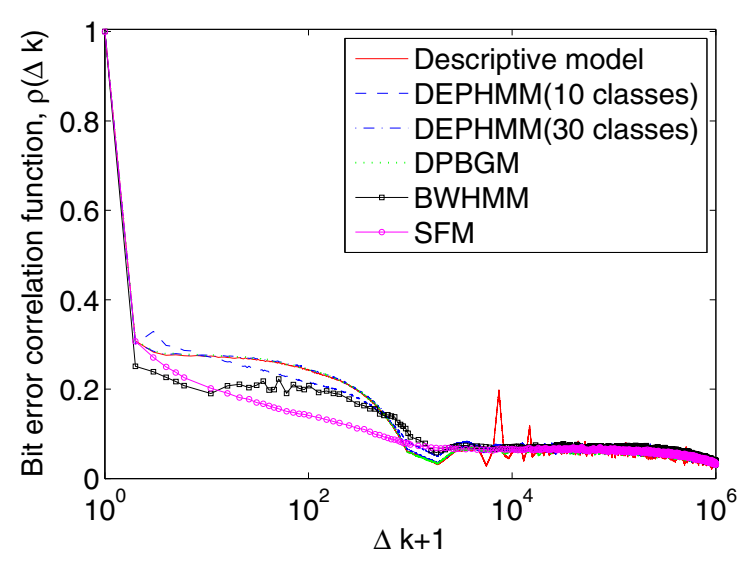

Fig. 7. Bit error correlation functions of the descriptive model obtained from the EGPRS system and different generative models. 